croenvironment. However, we agree with Arnaud et al. that the exact molecular basis of T-cell activation, especially in the case of psoriasis, needs to be addressed in further studies.

Borriello and De Palma address the important aspect of clinical decision making. Historically, the diagnosis of atopic eczema and the hyperIgE syndrome has been based on clinical phenotyping and laboratory findings. Patient 2 did not fulfill these criteria for the hyper-IgE syndrome. ${ }^{3}$ However, as Borriello and De Palma state, both diseases are heterogeneous, and the clinical presentations overlap. With increasing knowledge of altered molecular pathways and distinct genetic mutations, several disease subgroups may be identified. Such a process has been observed in the field of cancer, in which detailed knowledge of the pathogenesis of diseases such as melanoma ${ }^{4}$ is the basis for an individualized molecular therapy. Interestingly, no patient with atopic eczema in our article was filaggrin-deficient, which could indicate a "T-cell-driven" or "antigen-dependent" type of atopic eczema. Certainly, the way to pro- ceed is to identify the precise molecular and genetic alterations of individuals rather than groups of patients.

Stefanie Eyerich, Ph.D.

Helmholtz Center Munich

Munich, Germany

kilian.eyerich@Irz.tum.de

Anna T. Onken, M.D.

Kilian Eyerich, M.D., Ph.D.

Technische Universität Munich

Munich, Germany

Since publication of their article, the authors report no further potential conflict of interest.

1. Traidl-Hoffmann C, Mariani V, Hochrein H, et al. Pollenassociated phytoprostanes inhibit dendritic cell interleukin-12 production and augment $\mathrm{T}$ helper type 2 cell polarization. J Exp Med 2005;201:627-36. [Erratum, J Exp Med 2005;201:1347.]

2. Eyerich S, Eyerich K, Pennino D, et al. Th22 cells represent a distinct human $\mathrm{T}$ cell subset involved in epidermal immunity and remodeling. J Clin Invest 2009;119:3573-85.

3. Grimbacher B, Holland SM, Gallin JI, et al. Hyper-IgE syndrome with recurrent infections - an autosomal dominant multisystem disorder. N Engl J Med 1999;340:692-702.

4. Curtin JA, Fridlyand J, Kageshita T, et al. Distinct sets of genetic alterations in melanoma. N Engl J Med 2005;353:213547.

\title{
Coagulopathy of Chronic Liver Disease
}

TO THE EDITOR: In their review article, Tripodi and Mannucci (July 14 issue) ${ }^{1}$ present data that suggest a procoagulant imbalance in cirrhosis. Resistance to anticoagulation that is mediated by thrombomodulin ${ }^{2}$ and an increased relative risk of venous thromboembolism ${ }^{3}$ indicate that patients with cirrhosis do not undergo anticoagulation because of their increased prothrombin time. Consequently, the rationale to administer freshfrozen plasma to correct the prothrombin time before invasive procedures is debated.

One of the invasive procedures that is most frequently performed in patients with decompensated cirrhosis is diagnostic or large-volume paracentesis. Even in patients with elevated values for the international normalized ratio, bleeding after paracentesis occurs in a very low percentage of patients, ${ }^{4}$ and there is no evidence that a prolonged prothrombin time is associated with a risk of bleeding after this procedure. ${ }^{5}$ These findings indicate that a prolonged prothrombin time is not associated with a clinically relevant hypocoagulable state in patients with cirrhosis. Therefore, avoiding the administration of fresh-frozen plasma before paracentesis in patients with cirrhosis should be considered, since the risks and costs may exceed the real benefit for the patient.

Andrea De Gottardi, M.D., Ph.D.

University of Bern

Bern, Switzerland

andrea.degottardi@gmail.com

No potential conflict of interest relevant to this letter was reported.

1. Tripodi A, Mannucci PM. The coagulopathy of chronic liver disease. N Engl J Med 2011;365:147-56.

2. Tripodi A, Primignani M, Chantarangkul V, et al. An imbalance of pro- vs anti-coagulation factors in plasma from patients with cirrhosis. Gastroenterology 2009;137:2105-11.

3. S申gaard KK, Horváth-Puhó E, Grønbaek H, Jepsen P, Vilstrup H, Sфrensen HT. Risk of venous thromboembolism in patients with liver disease: a nationwide population-based casecontrol study. Am J Gastroenterol 2009;104:96-101.

4. Pache I, Bilodeau M. Severe haemorrhage following abdominal paracentesis for ascites in patients with liver disease. Aliment Pharmacol Ther 2005;21:525-9.

5. De Gottardi A, Thévenot T, Spahr L, et al. Risk of complications after abdominal paracentesis in cirrhotic patients: a prospective study. Clin Gastroenterol Hepatol 2009;7:906-9.

TO THE EDITOR: Tripodi and Mannucci indicate that anticoagulation should be commonly used 
in patients with cirrhosis who have portal-vein thrombosis caused by a procoagulant imbalance. However, a prospective study has shown that the only independent predictor of portal-vein thrombosis in liver cirrhosis is a reduced velocity in portal-vein flow. ${ }^{1}$ This finding suggests that the most critical issue for the treatment of portal-vein thrombosis in patients with cirrhosis should be how to improve portal-vein flow. In this situation, the benefit of the placement of a transjugular intrahepatic portosystemic shunt (TIPS) is prominent, because the procedure not only recanalizes the thrombosed portal vein by means of endovascular manipulation but also increases the portal bloodflow velocity by creating a portosystemic shunt. ${ }^{2,3}$ Until now, data for 243 patients with cirrhosis and portal-vein thrombosis who underwent TIPS have been reported in 10 case series (unpublished data). By contrast, the use of anticoagulation for portalvein thrombosis in patients with cirrhosis has been reported in only three case series. Notably, anticoagulation is often used in patients with partial portal-vein thrombosis but rarely in those with complete portal-vein thrombosis or portal cavernoma. ${ }^{4}$ In addition, questions remain about the type, dose, and duration of anticoagulation.

Xingshun Qi, M.D.

Guohong Han, M.D.

Daiming Fan, M.D., Ph.D.

Fourth Military Medical University

Xi'an, China

guohhan@126.com

No potential conflict of interest relevant to this letter was reported.

1. Zocco MA, Di Stasio E, De Cristofaro R, et al. Thrombotic risk factors in patients with liver cirrhosis: correlation with MELD scoring system and portal vein thrombosis development. J Hepatol 2009;51:682-9.

2. Qi X, Han G, Fan D. The preferable treatment for cirrhotic portal vein thrombosis: anticoagulation or transjugular intrahepatic portosystemic shunt? Hepatology 2010;51:713-4.

3. Han G, Qi X, He C, et al. Transjugular intrahepatic portosystemic shunt for portal vein thrombosis with symptomatic portal hypertension in liver cirrhosis. J Hepatol 2011;54:78-88.

4. Qi X, Han G, Wu K, Fan D. Anticoagulation for portal vein thrombosis in cirrhosis. Am J Med 2010;123(9):e19-e20.

TO THE EDITOR: A procoagulant state is detected in liver cirrhosis and may be responsible for an enhanced rate of vein thrombosis. Tripodi and Mannucci suggest that an imbalance between increased values of factor VIII and reduced natural anticoagulants such as protein $\mathrm{C}$ may account for the procoagulant state. Enhanced circulating lev- els of von Willebrand factor are suggested to impair factor VIII clearance, but the underlying mechanism is not fully clarified. We have previously shown that endotoxemia plays a role, since it is enhanced in patients with cirrhosis and may release von Willebrand factor from endothelial cells. ${ }^{1}$ Interestingly, endotoxemia may elicit a procoagulant state with an alternative mechanism, since it enhances the expression of tissue factor (with ensuing clotting activation) in monocytes obtained from patients with cirrhosis. ${ }^{2}$ This effect is more pronounced in the portal circulation of patients with cirrhosis in whom endotoxemia could be a trigger for thrombosis. ${ }^{3}$ The treatment of such patients with nonabsorbable antibiotics resulted in parallel reduction of endotoxemia, von Willebrand factor, and clotting activation, ${ }^{4}$ which suggests that this approach may lower the procoagulant state in patients with cirrhosis.

Francesco Violi, M.D.

Domenico Ferro, M.D.

Stefania Basili, M.D.

Sapienza University of Rome

Rome, Italy

francesco.violi@uniromal.it

No potential conflict of interest relevant to this letter was reported.

1. Ferro D, Quintarelli C, Lattuada A, et al. High plasma levels of von Willebrand factor as a marker of endothelial perturbation in cirrhosis: relationship to endotoxemia. Hepatology 1996;23: 1377-83.

2. Saliola M, Lorenzet R, Ferro D, et al. Enhanced expression of monocyte tissue factor in patients with liver cirrhosis. Gut 1998; 43:428-32.

3. Violi F, Ferro D, Basili S, et al. Ongoing prothrombotic state in the portal circulation of cirrhotic patients. Thromb Haemost 1997;77:44-7.

4. Violi F, Ferro D, Basili S, et al. Association between lowgrade disseminated intravascular coagulation and endotoxemia in patients with liver cirrhosis. Gastroenterology 1995;109: 531-9.

THE AUTHORS REPLY: We agree with De Gottardi that there is no need for plasma prophylaxis in patients with an abnormal prothrombin time who undergo paracentesis. ${ }^{1} \mathrm{~A}$ rescue strategy (i.e., wait and see if bleeding occurs) is more reasonable, although evidence stemming from controlled clinical trials is needed.

Like Qi et al., we had indeed emphasized in our review article that reduced flow velocity is one of the determinants of portal-vein thrombosis. ${ }^{2}$ However, reduced flow velocity, in addition to vessel-wall abnormalities and procoagulant im- 
balance (i.e., reduced levels of protein $\mathrm{C}$ and increased factor VIII), are also mechanistic factors for Virchow's triad, which leads to an increased risk of venous thrombosis. Therefore, we believe there is a rationale for using anticoagulation in patients with portal-vein thrombosis. Furthermore, TIPS, although effective for the treatment of portal-vein thrombosis, is an invasive procedure. ${ }^{3}$ Therefore, we consider TIPS a second-line therapeutic option to be implemented only when there are concomitant complications related to portal hypertension or when thrombosis expands despite anticoagulant therapy.

As pointed out by Violi et al., ${ }^{4}$ the occurrence of endotoxemia might play a role in the perturbation of hemostasis in end-stage liver disease through alterations of fibrinolysis or the occurrence of disseminated intravascular coagulation. However, the role that is played by the above conditions cannot be easily ascertained in the clinical context, owing to the lack of relatively simple global laboratory tests that truly reflect the conditions operating in vivo. For instance, increased levels of prothrombin fragment $\mathrm{F} 1+2$ or D-dimer, which are regarded by Violi et al. ${ }^{4}$ as indexes of coagulation activation, may instead be the consequence of the decreased clearance of these peptides from the circulation owing to impaired liver function rather than to ongoing coagulation activation. Hence, the association of these abnormali- ties with bleeding or thrombotic complications in end-stage liver disease cannot be easily shown. Finally, the question of whether the administration of nonabsorbable antibiotics truly helps to limit the procoagulant imbalance in end-stage liver disease, as suggested by Violi et al., should be more thoroughly investigated in prospective, randomized clinical trials. However, we maintain that antibiotics are less likely than anticoagulants to affect the plasma procoagulant imbalance because of the reduced levels of protein $\mathrm{C}$ and increased factor VIII that are distinctive features of chronic liver disease.

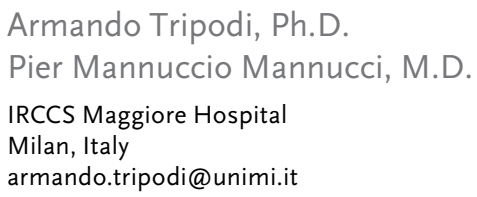

Since publication of their article, the authors report no further potential conflict of interest.

1. De Gottardi A, Thévenot T, Spahr L, et al. Risk of complications after abdominal paracentesis in cirrhotic patients: a prospective study. Clin Gastroenterol Hepatol 2009;7:906-9.

2. Zocco MA, Di Stasio E, De Cristofaro R, et al. Thrombotic risk factors in patients with liver cirrhosis: correlation with MELD scoring system and portal vein thrombosis development. J Hepatol 2009;51:682-9.

3. Qi X, Han G, Fan D. The preferable treatment for cirrhotic portal vein thrombosis: anticoagulation or transjugular intrahepatic portosystemic shunt? Hepatology 2010;51:713-4.

4. Violi F, Ferro D, Basili S, et al. Association between low-grade disseminated intravascular coagulation and endotoxemia in patients with liver cirrhosis. Gastroenterology 1995;109:531-9.

\section{The Avastin Story}

TO THE EDITOR: Recently, the Food and Drug Administration (FDA) held an administrative hearing regarding the proposal to withdraw accelerated approval from Avastin (bevacizumab) for metastatic breast cancer. I served as a member of the Oncologic Drugs Advisory Committee (ODAC) at that meeting. Two recent online Perspective articles, by Carpenter et al. ${ }^{1}$ and D'Agostino, ${ }^{2}$ addressed the use of surrogate end points and the regulatory and political issues surrounding this hearing. But neither article covered a basic, patient-focused theme that we, the oncologists on the panel, considered: that of giving hope - but not false hope.
Avastin received accelerated approval from the FDA for metastatic breast cancer in 2008 on the basis of a study in which patients who were treated with Avastin, in combination with paclitaxel, had a progression-free survival advantage of 5.5 months over paclitaxel alone but no overall survival advantage. ${ }^{3}$ Toxic effects were substantial, but this interim end point suggested real clinical benefit. Unfortunately, in what were supposed to be confirmatory studies enrolling almost 2000 women, progression-free survival was shorter than in the initial study, and no qualityof-life or survival advantage could be shown for Avastin-containing regimens. 